\title{
Tratamento de EFLUENTES: UMA INVESTIGAÇÃo DE FILTROS ANAERÓBIOS através da proposta do Professor Cynamon
}

\section{WEBERTON DANTAS DE SOUSA ${ }^{1 *}$, LUIS FERNANDO DE OLIVEIRA COELHO ${ }^{1}$, ANDRÉA MARIA BRANDÃO MENDES DE OLIVEIRA ${ }^{2}$}

\author{
${ }^{1}$ Mestrandos em Sistemas Agroindustriais/Universidade Federal de Campina Grande. \\ ${ }^{2}$ Unidade Acadêmica de Ciências e Tecnologia Ambiental (UACTA/CCTA)/ Universidade Federal de Campina Grande. \\ * Autor para correspondência: weberton250@hotmail.com
}

Recebido em 11 de setembro de 2017. Aceito em 05 de setembro de 2018. Publicado em 28 de dezembro de 2018.

REsumo - É essencial em uma sociedade cada vez mais preocupada com as questões ambientais o uso de técnicas e procedimentos que venham a tratar o efluente gerado em suas atividades de modo a garantir a qualidade ambiental. Utilizar meio eficientes e sustentáveis é necessário para atender as características do meio e o atendimento a maior parte da população que carecem de sistemas de tratamento de esgotos. Nessa perspectiva, os filtros anaeróbios se apresentam como uma das alternativas na solução destes problemas, uma vez que vários estudos demonstram sua eficiência e o baixo custo de sua construção e manutenção. Uma variação na utilização deste filtro foi proposta por Cynamon em 1986, com uma série de três filtros em fluxos ascendente, descendente e ascendente, respectivamente. Este trabalho teve por objetivo testar filtros anaeróbios propostos por Cynamon para tratamento de efluentes oriundos de atividades domésticas. O esgoto bruto foi tratado em dois sistemas de filtros adaptados, um com fluxos ascendentes e outro com fluxos descendentes. O efluente bruto e tratado foram submetidos a testes de $\mathrm{pH}$, cor, turbidez, temperatura, DQO e coliformes totais e termotolerantes. Foi possível observar que os filtros ascendentes obtiveram melhores resultados quanto as variáveis observadas se comparados ao sistema descendente.

Palavras-chave: Tratamento de Esgotos Sanitários; Filtros anaeróbios; Prof. Cynamon.

\section{Tratamiento de efluentes: una inVEStigación de filtros anaeróbicos a través de la Propuesta del Professor Cynamon}

REsumen - Es esencial en una sociedad cada vez más preocupada con cuestiones ambientales el uso de técnicas y procedimientos que tratan el efluente generado en sus actividades para asegurar la calidad ambiental. Es necesario utilizar recursos eficientes y sostenibles para atender a las características del entorno y la atención a una gran parte de la población que carece de sistemas de tratamiento de aguas residuales. En esta perspectiva, los filtros anaerobios se presentan como una de las alternativas en la solución de estos problemas, ya que varios estudios demuestran su eficacia y bajo costo de su construcción y mantenimiento. Cynamon en 1986 propuso una variación en el uso de este filtro con una serie de tres filtros en flujos ascendente, descendente y ascendente, respectivamente. Este estudio pretende probar los filtros anaerobios propuestos por Cynamon para el tratamiento de efluentes derivados de actividades domésticas. Las aguas residuales brutas fueron tratadas en dos sistemas de filtros adaptados, uno con el aumento de los flujos y otro con flujos descendentes. Los efluentes bruto y tratado fueron sometidos a pruebas de $\mathrm{pH}$, color, turbidez, temperatura, DQO, así como los coliformes totales y los termotolerantes. Se observó que los filtros ascendentes obtuvieron mejores resultados en cuanto a las variables observadas en comparación con el sistema descendente.

Palabras clave: Tratamiento de aguas residuales domésticas; Filtros anaeróbios; Prof. Cynamon 


\section{Effluent treatment: anaerobic filters research through the proposal of Professor Cynamon}

AвSTRACT - It is essential in a society increasingly concerned with environmental issues using techniques and procedures that will treat the effluent generated in its activities to ensure environmental quality. Using efficient and sustainable means is necessary to meet the characteristics of the environment and the assistance to the majority of the population lacking sewage treatment systems. In this perspective, anaerobic filters are presented as one of the alternatives in solving these problems, since several studies show its efficiency and the low cost of maintenance. A variation on the use of this filter was proposed by Cynamon in 1986, with a series of three filters in ascending, descending and ascending streams, respectively. This work aimed to test anaerobic filters proposed by Cynamon to treat effluents from domestic activities. The raw sewage was treated in two adapted filter systems, one with upstream flows and the other with downstream streams. The crude and treated effluent were submitted to tests of $\mathrm{pH}$, color, turbidity, temperature, COD and total and thermotolerant coliforms. It was possible to observe that the ascending filters obtained better results than the observed variables when compared to the descending system.

Keywords: Sewage Treatment; Anaerobic filters; Prof. Cynamon.

\section{INTRODUÇÃO}

von Sperling e Chernicharo (1996) apontam como causa principal da carência de sistemas de tratamento de esgotos a falta de investimentos concretos no setor, que além de deteriorar as condições adequadas de funcionamento de estações de tratamento já existentes impedem a construção de novas e que, pela burocracia, muitas vezes ao serem concluídas já não são suficientes para atender a uma população que cresce cada vez mais. Essa observação de von Sperling e Chernicharo apesar de distar 20 anos parece ser recente, mesmo levando em conta os avanços no setor nos últimos 10 anos.

Esta situação acaba por aumentar os problemas com relação a um meio ambiente saudável e adequado para usufruto de todos. É apontado como uma das soluções ao problema o desenvolvimento e implantação de sistemas de tratamento de baixo custo de construção e manutenção, que são compatíveis com as condições financeiras da maioria das cidades brasileiras, mas que sejam capazes de realizar um tratamento adequado. Segundo Santos (2012) "entende-se por sistemas simples aqueles que empregam métodos naturais, que são pouco mecanizados, têm baixo custo de construção e operação, além de serem viáveis e sustentáveis”. O campo do saneamento, especialmente o tratamento de efluentes no Brasil e mesmo no mundo, considerando o conceito de pegada ambiental carece de tecnologia eficiente, de baixo custo de instalação e manutenção. Nesse sentido o uso de filtros anaeróbios, com fluxo ascendente ou descendente, propulsionados pela gravidade gozam de bom aceite.

De acordo com a Associação Brasileira de Normas Técnicas (1993), o filtro anaeróbio é uma "unidade destinada ao tratamento de esgoto, mediante afogamento do meio biológico filtrante". Conforme Castro e Silva (2014) "os filtros anaeróbios são reatores biológicos formados por um conjunto de partes imóveis de material inerte, envoltos de microrganismos não aeróbios na forma de biofilme pelo qual o efluente é depurado".

Um exemplo deste tipo de tratamento é o proposto por Cymamon (1986), onde se propõe uma série de três filtros com fluxos ascendente, descendente e ascendente seguidos por uma fase de polimento com filtro de areia. De acordo com Roque e Mello Júnior (2014), “o processo assemelha-se aos filtros anaeróbios comuns, com a vantagem de praticamente dividir o tratamento em três fases, dando tempo necessário para uma máxima atuação dos microrganismos". Além disso, sua construção é igual aos filtros comuns, sendo, portanto, de fácil operação e manutenção. O objetivo do trabalho foi testar filtros anaeróbios propostos por Cynamon (1986) para tratamento de efluentes oriundos de atividades domésticas. 


\section{Material e Métodos}

As duas séries de filtros anaeróbios foram construídos em fluxos totalmente ascendentes e totalmente descendentes, sendo elas independentes entre si, dimensionados segundo Associação Brasileira de Normas Técnicas (1997), que trata dos tanques sépticos, unidades de tratamento complementar e disposição final dos efluentes líquidos. Segundo esta norma, o volume dos filtros é função do número de contribuintes $(\mathrm{N})$, da contribuição em litros/dia (C) e do tempo de detenção hidráulica (T), onde a contribuição varia de acordo com o tipo de ocupação predial da fonte geradora e o tempo de detenção hidráulica de acordo com a vazão e a temperatura local.

Conforme o especificado na Associação Brasileira de Normas Técnicas (1997) para dimensionamento de filtros anaeróbios e considerando o Bloco II de aulas da UFCG/CCTA, fonte do efluente bruto, como edifício público com ocupantes temporários, a contribuição de despejo por habitante é de $50 \mathrm{~L} / \mathrm{d}$ segundo a referida norma para esse tipo de ocupação. O sistema de filtros proposto por Cynamon foi desenvolvido para atender a uma população de 480 pessoas, caracterizando a seguinte vazão diária $(\mathrm{Vd})$.

$$
\begin{gathered}
\mathrm{Vd}=\mathrm{N} \times \mathrm{C} \\
\mathrm{Vd}=480 \times 50 \\
\mathrm{Vd}=24000 \mathrm{~L} / \mathrm{d}
\end{gathered}
$$

(Equação 1)

Para esta vazão diária calculada e a temperatura média do mês mais frio acima de $25^{\circ} \mathrm{C}$ na cidade de Pombal, onde está situada a mencionada instituição de ensino, o tempo de detenção hidráulica é de 0,5 dias conforme o especificado na norma. Assim o volume dos filtros é dado por:

$$
\begin{gathered}
\mathrm{Vu}=1,6 \times \mathrm{N} \times \mathrm{C} \times \mathrm{T} \\
\mathrm{Vu}=1,6 \times 480 \times 50 \times 0,5 \\
\mathrm{Vu}=19,2 \mathrm{~m}^{3}
\end{gathered}
$$

Este volume foi transformado em $0,019 \mathrm{~m}^{3}$ em escala reduzida de 1:10 para a construção dos filtros em série em escala piloto. Como o filtro foi desenvolvido no formato retangular o volume então é determinado como sendo igual ao produto da altura, largura e comprimento. As dimensões de comprimento (c) e largura (l) da caixa plástica utilizada foram de $0,45 \mathrm{~m}$ e $0,3 \mathrm{~m}$ respectivamente, logo altura de meio filtrante (a) foi então determinada através do seguinte cálculo:

$$
\begin{gathered}
\mathrm{Vu}=\mathrm{c} \times \mathrm{a} \times \mathrm{l} \\
0,019=0,45 \times \mathrm{a} \times 0,3 \\
\mathrm{a}=0,14 \mathrm{~m}
\end{gathered}
$$

(Equação 3)

Os filtros anaeróbios e de polimento em caixa de areia foram construídos em escala piloto por meio de caixas plásticas. O meio filtrante utilizado foi a pedra britada n 2 conforme a Associação Brasileira de Normas Técnicas (1993). A confecção em materiais alternativos às construções de alvenaria permite a redução do custo e maior comodidade, e seu uso é recomendado Associação Brasileira de Normas Técnicas (1997), unidades de tratamento complementar e disposição final dos efluentes líquidos, onde recomenda-se para a construção de filtros anaeróbios materiais como concreto armado, plástico e vidro de alta resistência.

Para passagem do efluente construiu-se malhas em tubos de PVC Ø20mm perfurados. As malhas possuem comprimentos de $20 \mathrm{~cm}$ nos filtros de brita $\mathrm{n}^{\circ} 2$ conforme a Associação Brasileira de Normas Técnicas (2005) e $10 \mathrm{~cm}$ nos filtros de polimento devido ao seu menor comprimento. Segundo a norma utilizada para a construção, a colocação deve ser feita horizontalmente e no sentido do maior ângulo. Os furos feitos na canalização foram igualmente espaçados em $2 \mathrm{~cm}$ seguindo a regra da Associação Brasileira de Normas Técnicas (1997). Foram colocadas duas malhas por caixa, uma na parte superior e outra na parte inferior em ambos os sistemas, sendo uma com função de aspergir e outra de coletar. 
O projeto foi instalado no Laboratório de Análises de Água da Universidade Federal de Campina Grande - Campus Pombal. O esgoto bruto aplicado era proveniente da Central de Aulas II apresentando características tipicamente domésticas previamente tratadas em tanque séptico. As amostras empregadas nas análises foram obtidas em três pontos igualmente selecionados nos dois sistemas, sendo antes da entrada do líquido nos filtros, entre o terceiro filtro e a caixa de polimento e após passagem total pelos sistemas. Apenas a temperatura foi obtida em todos os filtros de cada sistema.

As amostras foram coletadas de forma pontual e representadas por EB (Efluente Bruto), S1F1 (Filtro 1 do Sistema Descendente), S1F2 (Filtro 2 do Sistema Descendente) S1F3 (Filtro 3 do Sistema Descendente), S1P (Filtro de Polimento do Sistema Descendente), S2F1 (Filtro 1 do Sistema Ascendente), S2F2 (Filtro 2 do Sistema Ascendente), S2F3 (Filtro 3 do Sistema Ascendente), S2P (Filtro de Polimento do Sistema Ascendente), por um período de 15 dias a partir da maturação, resultando em uma média de 30 coletas para análises físicas e químicas e 3 coletas para microbiológicas. Quanto a temperatura, as análises foram efetuadas quatro vezes ao dia durante 15 dias.

As amostras foram submetidas à análises físicas, químicas e microbiológicas através das variáveis pH, Cor, Turbidez, Temperatura, DQO (Demanda Química de Oxigênio), Coliformes Totais (CT) e Termotolerantes (Cter). As quatro primeiras variáveis seguiram as metodologias do fabricante. Para demanda química de oxigênio e coliformes foi empregada a metodologia Brasil (2009).

\section{Resultados e Discussão}

Após a construção, os dois sistemas passaram a receber o efluente bruto. As amostras foram coletadas e submetidas a análises físicas, químicas e microbiológicas. Os dados observados nas variáveis cor aparente, turbidez, pH estão dispostos, em média, na Tabela 1 abaixo.

Tabela 1 - Resultados médios das análises de $\mathrm{pH}$, cor aparente e turbidez nos pontos de coletas dos sistemas.

\begin{tabular}{cccc}
\hline Amostras & $\mathrm{pH}$ & Cor (UC) & Turbidez (NTU) \\
\hline EB & 8,56 & 286,71 & 27,85 \\
S1F3 & 8,54 & 266 & 24,85 \\
S1P & 8,53 & 261,42 & 21 \\
S2F3 & 8,60 & 74,37 & 10,42 \\
S2P & 8,82 & 67,48 & 9,85 \\
\hline
\end{tabular}

Neste trabalho, o pH dos esgotos variou entre 8,53 e 8,82 nos dois sistemas. Para disposição final o efluente após tratamento apresenta pH dentro dos padrões exigidos pela Brasil (2011) que variam de 5 a 9.

Os dados referentes à cor apresentavam-se elevados em ambos os sistemas, porém no fluxo ascendente houveram as maiores reduções nos teores dessa variável em torno de $76,46 \%$, se comparados a entrada de afluente e a saída pós-tratamento. Nos filtros descendentes a proporção foi de apenas 8,20\%.

A Turbidez também apresentou os melhores resultados no sistema ascendente quando a variação na redução foi de 64,63\% se comparado ao descendente onde foi de 24,60\%. Embora não sejam variáveis exigidas por lei, de acordo com Jordão e Pessoa (2014), estas assumem importância particular quando o efluente tratado é lançado em corpos d'água onde os aspectos estéticos são importantes. Importante ressaltar que a média de redução em ambas as variáveis foram feitas naqueles em que foi possível calcular entre o valor da amostra bruta e o valor após o tratamento completo.

Durante o período de análises foram verificados vazamentos que possivelmente influenciaram no tempo de detenção hidráulica de ambos os sistemas fazendo com que aumentasse nos filtros ascendentes e diminuísse nos filtros descendentes, o que pode ter vindo a causar as discrepâncias entre um sistema e outro. 
A Temperatura em ambos os sistemas foi verificada em todos os filtros para garantir uma maior homogeneidade. Este é um fator importante em filtros anaeróbios uma vez que estão relacionados com a eficiência de remoção da Matéria Orgânica (DBO - Demanda Bioquímica de Oxigênio e DQO). Os resultados observados estão descritos na Tabela 2.

Tabela 2- Temperatura $\left({ }^{\circ} \mathrm{C}\right)$ média no período de coleta em todo o sistema.

\begin{tabular}{ccccc}
\hline & \multicolumn{5}{c}{ Horários } \\
\hline Sistemas & $07: 00$ & $11: 00$ & $13: 00$ & $17: 00$ \\
\hline EB & 28,0 & 31,0 & 33,2 & 34,8 \\
S1F1 & 28,0 & 30,7 & 34,9 & 35,3 \\
S1F2 & 28,0 & 30,7 & 34,6 & 35,2 \\
S1F3 & 28,2 & 30,9 & 35,0 & 36,1 \\
S1P & 28,3 & 32,4 & 35,6 & 35,4 \\
S2F1 & 28,4 & 30,6 & 34,6 & 35,6 \\
S2F2 & 28,5 & 31,5 & 34,6 & 35,5 \\
S2F3 & 28,4 & 31,0 & 34,8 & 35,3 \\
S2P & 28,2 & 31,1 & 34,6 & 35,0 \\
\hline
\end{tabular}

Segundo Jordão e Pessoa (2014), uma faixa adequada de temperatura para a maior eficiência da ação dos microrganismos está entre 25 a $35^{\circ} \mathrm{C}$. Importante ressaltar que abaixo de $15^{\circ} \mathrm{C}$ a digestão anaeróbia não se processa, afetando a eficiência dos filtros propostos por Cynamon.

Neste estudo as temperaturas nos sistemas ascendentes e descendentes variaram entre $28^{\circ} \mathrm{C}$ pela manhã até $36,1^{\circ} \mathrm{C}$ no período da tarde, próximos a faixa especificada. Segundo Lins (2010) essa faixa caracteriza-se como mesófila $\left(15^{\circ} \mathrm{C}\right.$ a $\left.45^{\circ} \mathrm{C}\right)$ e nesta a digestão anaeróbia se desenvolve bem em temperaturas de $30^{\circ} \mathrm{C}$ a $40^{\circ} \mathrm{C}$. É válido ressaltar a necessidade de que se opere o tratamento em condições de pouca variação de temperatura. Quanto ao lançamento, o efluente após passagem nos filtros está dentro do que preconiza a Brasil (2011) que estabelece um valor inferior a $40^{\circ} \mathrm{C}$ ou não aumentar a Temperatura do corpo d'água na zona de mistura em até $3^{\circ} \mathrm{C}$.

Outro importante fator a ser analisado na verificação da eficiência dos filtros anaeróbios propostos por Cynamon é a DQO, que permite avaliar a redução da matéria orgânica dos esgotos. A presença excessiva desta nos efluentes ao serem lançados nos corpos hídricos reduz a passagem de luz e de OD (Oxigênio Dissolvido). A Tabela 3 demonstra a DQO observada durante o tratamento nos filtros em escala piloto.

Tabela 3 - DQO nos pontos de coleta dos sistemas.

\begin{tabular}{cccc}
\hline Amostras & \multicolumn{3}{c}{ DQO } \\
\hline & Coleta 1 & Coleta 2 & Coleta 3 \\
\cline { 2 - 4 } EB & 158,10 & 167,88 & 227,72 \\
S1F3 & 118,57 & 138,33 & 120,55 \\
S1P & 81,02 & 167,88 & 128,45 \\
S2F3 & 59,28 & 69,16 & 77,07 \\
S2P & 49,40 & 65,21 & 73,12 \\
\hline
\end{tabular}

De acordo com a Associação Brasileira de Normas Técnicas (1997), a faixa de eficiência dos filtros anaeróbios em conjunto com tanque séptico, como o ocorrido neste trabalho, a DQO varia de 40 a $70 \%$ de acordo com a temperatura. $\mathrm{O}$ limite inferior é para a temperatura abaixo de $15^{\circ} \mathrm{C}$ e o superior para temperatura acima de $25^{\circ} \mathrm{C}$. 
Baseado nisso, o filtro CYNAMON de fluxo ascendente obteve os valores mais próximos do estabelecido para a temperatura acima de $25^{\circ} \mathrm{C}$, já que teve em média $65,93 \%$ de redução de DQO se comparado aos 30,78\% do filtro de fluxo descendente.

É possível verificar na Tabela 3 um aumento nos valores de DQO no S1P nas Coletas 2 e 3, isto pode ter ocorrido provavelmente em virtude da troca de leito filtrante. Esta troca ocorreu devido a uma quebra do sistema montado, sendo necessária a remontagem do filtro de polimento. Por este motivo, a areia foi completamente substituída. Mesmo com essa alteração, Machado e Chernicharo (1997) também observaram em seu trabalho uma melhor eficiência nos filtros ascendentes se comparados aos descendentes.

De acordo com Sylvestre (2013) “durante o processo de tratamento de águas residuárias é importante que se faça a contagem padrão de bactérias, visto que o resultado permite avaliar a eficiência das várias etapas do processo". A Tabela 4 mostra os resultados obtidos na análise do efluente do Bloco II de aulas do CCTA/UFCG.

Tabela 4 - Resultados das análises microbiológicas nos pontos de coleta dos sistemas.

\begin{tabular}{|c|c|c|c|c|}
\hline \multirow[b]{2}{*}{ Parâmetros } & \multicolumn{4}{|c|}{ Coletas } \\
\hline & Amostras & Coleta 1 & Coleta 2 & Coleta 3 \\
\hline \multirow{5}{*}{ CT } & $\mathrm{EB}$ & 1100 & $>1100$ & $>1100$ \\
\hline & S1F3 & 1100 & 240 & $>1100$ \\
\hline & S1P & 93 & Ausente & 1100 \\
\hline & $\mathrm{S} 2 \mathrm{~F} 3$ & Ausente & 1100 & 240 \\
\hline & S2P & Ausente & 3,6 & 240 \\
\hline \multirow{5}{*}{ Cter } & $\mathrm{EB}$ & 240 & 240 & $>1100$ \\
\hline & S1F3 & 1100 & 93 & 1100 \\
\hline & S1P & 93 & 1100 & 1100 \\
\hline & $\mathrm{S} 2 \mathrm{~F} 3$ & Ausente & 240 & 93 \\
\hline & S2P & Ausente & 0 & 240 \\
\hline
\end{tabular}

Analisando os dados da Tabela 4 foi possível observar a redução na presença de Ct e Cter com relação ao efluente bruto no filtro proposto por Cynamon em fluxo ascendente, embora na Coleta 3 tenha havido elevação entre o S2F3 e S2P, indicando possível contaminação. No fluxo descendente também foi verificado redução, mesmo que em menor grau, embora na Coleta 2 tenha sido constatado um aumento significativo em relação ao S1P, provavelmente em função da troca do leito filtrante, já explicado anteriormente.

Os filtros anaeróbios assim como reatores $U A S B$ e tanque sépticos são exemplos de reatores anaeróbios. Segundo Chernicharo et al. (2001) "a remoção de coliformes nos reatores anaeróbios tem baixa eficiência, usualmente na ordem de uma unidade logarítmica”. Essa afirmação é possível ser verificada em testes realizados com filtros anaeróbios para redução de indicadores de contaminação (coliformes totais e fecais), onde os resultados não atingem os patamares desejáveis em comparação a outros processos de tratamento (YOUNG e MAC CARTY, 1969; PAULA JR.,1983; DENNIS JR. e JENNET, 1974 apud ROQUE e MELLO JÚNIOR, 2014).

\section{ConClusões E RECOMENDAÇÃo}

Em ambos os sistemas foi possível verificar diminuição nas variáveis analisadas, embora o filtro com fluxo ascendente apresentasse reduções mais significativas. Se levarmos em consideração a Associação Brasileira de Normas Técnicas (1997) este filtro está mais próximo do que é preconizado, principalmente em fatores como a DQO, onde sua eficiência foi de 65,93\% comparados aos 30,78\% do fluxo descendente. 
A Cor e Turbidez apresentaram reduções entre o terceiro filtro e o polimento, no descendente a redução foi de $1,72 \%$ e $15,49 \%$, respectivamente, enquanto no ascendente foram de $9,26 \%$ e 5,47\%. Foi possível constatar que os filtros de polimento mesmo com pequenas reduções foram importantes para a melhoria das variáveis observadas, embora não se tenha verificado o mesmo para a DQO no fluxo descendente possivelmente em virtude de troca do leito filtrante por ocorrência de quebra do sistema de polimento.

Embora fatores externos com relação à operação possam ter vindo a influenciar nos resultados, o fluxo ascendente apresentou melhor eficiência que o descendente, corroborando com o já observado em outro trabalho e com o próprio filtro proposto por Cynamon (1986), no qual dois dos três filtros eram ascendentes.

\section{REFERÊNCIAS}

ASSOCIAÇÃO BRASILEIRA DE NORMAS TÉCNICAS. 1993. NBR 7229: projeto, construção e operação de sistemas de tanques sépticos. Rio de Janeiro.

ASSOCIAÇÃO BRASILEIRA DE NORMAS TÉCNICAS. 1993. NBR 7225: materiais de pedra e agregados naturais. Rio de Janeiro.

ASSOCIAÇÃO BRASILEIRA DE NORMAS TÉCNICAS. 1997. NBR 13969: tanques sépticos: unidades de tratamento complementar e disposição final dos efluentes líquidos: projeto, construção e operação. Rio de Janeiro.

BRASIL (Governo do), CONAMA. Resolução No 430, DE 13 DE MAIO DE 2011. Dispõe sobre as condições de lançamento de efluentes, complementa e altera a Resolução n 357, de 17 de março de 2005, do Conselho Nacional do Meio Ambiente - CONAMA. 9 p

BRASIL (Governo do). FUNDAÇÃO NACIONAL DE SAÚDE. 2009. Manual prático de análise de água. $3^{a}$ ed. rev. Brasilia: Ministério da Saúde. 144 p.

Castro e Silva P. 2014. Desempenho de um filtro anaeróbio de fluxo ascendente como unidade de tratamento para efluente da suinocultura. 102 f. Dissertação (Mestrado) - Universidade Federal de Lavras, Lavras-Minas Gerais.

Chernicharo CAL et. al. 2001. Introdução. IN: PROSAB- Programa de Pesquisa em saneamento Básico, Pós tratamento de efluentes de reatores anaeróbios, Brasília, p.19-34.

Dennis JR., Norman, Jennet C. 1974. Pharmaceutical waste treatment with an anaerobic filter Proc. In: 29th Industrial Waste Conference, Pardue. Anais... Pardue University. p.160-173.

Jordão EP, Pessoa CA. 2014. Tratamento de Esgotos Domésticos. 7. ed. Rio de Janeiro: SEGRAC.

Machado RMGurgel, Chernicharo CAL. 1997. Avaliação do desempenho de filtros anaeróbios utilizados para o polimento de efluentes de um reator UASB. In: Congresso Brasileiro de Engenharia Sanitária e Ambiental, 19., Foz do Iguaçu. Anais eletrônicos... Foz do Iguaçu, ABES, v. 1. p. 841-851.

Paula Jr. DR. 1985. Desempenho de um filtro anaeróbio piloto no tratamento de águas residuárias de uma indústria de conservas alimentícias. Dissertação de Mestrado apresentada à Escola de Engenharia de São Carlos, Universidade de São Paulo, jan. 123 p 
Roque OCC, Mello Jr HAA. 1999. Eficiência dos filtros anaeróbios tipo Cynamon no tratamento de esgotos. In: CONGRESSO DE BRASILEIRO DE ENGENHARIA SANITÁRIA E AMBIENTAL, 20, Rio de Janeiro. Anais eletrônicos... Rio de Janeiro, ABES, p.1-9.

Santos LR. 2012. Pós-tratamento de efluentes de filtro anaeróbio precedido de tanque séptico por filtros aeróbios intermitentes de areia. Dissertação (Mestrado em Ciência e Tecnologia Ambiental) - Universidade Estadual da Paraíba, Campina Grande.

Sylvestre SHZ. 2013. Desempenho de sistemas de reatores anaeróbios e aeróbio na remoção de coliformes e ovos de helmintos de águas residuárias de suinocultutra. Dissertação (Mestrado em Microbiologia Agropecuária) - Universidade Estadual Paulista, Jaboticabal.

von Sperling M, Chernicharo CAL. 1996. Tendências no tratamento simplificado de águas residuárias. Tópicos de relevância. In: SEMINÁRIO INTERNACIONAL TENDÊNCIAS NO TRATAMENTO SIMPLIFICADO DE ÁGUAS RESIDUÁRIAS. Belo Horizonte. Anais...Belo Horizonte, MG: DESA/UFMG, p. 3-11.

Young J, Mac Carty P. 1969. The anaerobic filter for waste treatment. Journal WPCF, 41 (5), p 160-173. 